\title{
Real-time capture of student reasoning while writing
}

\author{
Scott V. Franklin ${ }^{*}$ and Lisa M. Hermsen \\ Department of Physics, Rochester Institute of Technology, Rochester, New York 14623-5603, USA
}

(Received 6 May 2014; published 25 September 2014)

\begin{abstract}
We present a new approach to investigating student reasoning while writing: real-time capture of the dynamics of the writing process. Key-capture or video software is used to record the entire writing episode, including all pauses, deletions, insertions, and revisions. A succinct shorthand, "S notation," is used to highlight significant moments in the episode that may be indicative of shifts in understanding and can be used in followup interviews for triangulation. The methodology allows one to test the widespread belief that writing is a valuable pedagogical technique, which currently has little directly supportive research. To demonstrate the method, we present a case study of a writing episode. The data reveal an evolution of expression and articulation, discontinuous in both time and space. Distinct shifts in the tone and topic that follow long pauses and revisions are not restricted to the most recently written text. Real-time writing analysis, with its study of the temporal breaks and revision locations, can serve as a complementary tool to more traditional research methods (e.g., speak-aloud interviews) into student reasoning during the writing process.
\end{abstract}

DOI: 10.1103/PhysRevSTPER.10.020121

PACS numbers: 01.40.Fk, 01.40.gf

\section{INTRODUCTION}

Since the early 1970s Writing Across the Curriculum (WAC) and Writing in the Disciplines (WID) initiatives have theorized a link between language and learning. These posit writing as a higher order tool for content learning ("write-to-learn" [1-6]) or as a means for learning a discipline's epistemology [7], thus facilitating content learning. While these programs have succeeded at increasing the amount of writing in disciplinary courses, with associated gains in "learning to write," research into the specific claims of writing to learn have been thin, at best, with little supporting evidence. Research has been limited to local, classroom-based student writing assessments and (or) program-specific assessments [8-10]. Only recently have researchers [11] called for rigorous evaluation of the relationship between writing to learn and (1) content knowledge, (2) intellectual development, and (3) better disciplinary writing.

Ackerman [12] argued that "writing specialists tend to ignore the second half of the write-to-learn equationlearning and knowledge - and believe that the process and attributes of writing inevitably lead to learning." In an analysis of 35 studies that attempted to look empirically into the relation between writing and learning, he noted that such studies did not include more complex aspects of the writing process (e.g., multiple drafts) that mirror academic

\footnotetext{
*svfsps@rit.edu

Published by the American Physical Society under the terms of the Creative Commons Attribution 3.0 License. Further distribution of this work must maintain attribution to the author(s) and the published article's title, journal citation, and DOI.
}

writing. He concluded that, while writing likely does contribute and enrich the thought process, this is only true when writing is supported and valued within the specific context, a claim supported by Klein's finding that writing assignments did not produce a benefit in student performance on more superficial multiple-choice conceptual tests [13].

The use of writing in science environments also has a long history. Hein, in particular, has used student writing as both a research and learning tool [14,15]. More recently, Brookes and Etkina [16] have looked at the specific language and grammatical structures students use as they speak and write. Both have linked improved scores on traditional concept tests and increasingly sophisticated writing samples with reflective writing assignments. Similar efforts exist in primary school settings [17-22]. Nevertheless, a meta-analysis of 48 school-based writingto-learn programs [23] cited previous claims for writing as a strategy for enhancing learning but concluded that the whole body of research offered ambiguous conclusions. At best, a thorough review of research indicated only that "writing does appear to facilitate learning degree, under some conditions" [23].

Revision is commonly regarded as a central part of the writing process, in part because it enhances the final written document and also because it requires students to rework ideas [24]. Faigley and Witte [25] recognized the need to analyze revision in relation to text by distinguishing between text-based revisions that affect meaning and surface changes that do not. Schwartz [26] added to these observations by observing three major revision patterns: (1) language regeneration, (2) structural reformulation, and (3) content reassessment. Nevertheless, it has also been 
believed by composition scholars since at least 1983 [27] that new research methods would be essential to understand the revision process. While new studies of revision did appear, few, if any, focused on writing outside the composition classroom, or in writing in the disciplines [28]. More recently, research on revision practice has turned to computational practices, including the study of revision in multimodal writing tasks and the use of metadata in analysis of writing. Eye-tracking technologies have been used to analyze the reading and writing processes [29], in some instances combined with keystroke logging [30].

This article presents a methodology that allows capture and analysis of revisions on as small a scale as needed. Whereas version comparison (e.g., using the "track changes" feature in common word processors) highlight differences between drafts, key-capture analysis reveals revisions on the smallest scales, as words are typed, erased, and rewritten. This analysis captures the time evolution of a document, indicating the order in which words, sentences and even paragraphs are written. We present an example of the data this method enables from a student writing about an introductory physics topic, and highlight the new questions that may arise from this type of research.

\section{EXPERIMENTAL METHOD}

\section{A. Data acquisition and representation}

A variety of key-capturing software packages are commercially available, Inputlog [31], freely available to researchers, is popular among writing scholars (cf. Ref. [30]). There are ethical concerns with installing such software on individual personal computers and so we use laboratory computers, which require the subject's presence in the lab. This introduces an additional contextual element to the writing process. Equally, if not more, important to the researcher are techniques that present the data in forms that facilitate analysis. Here we describe S-notation, [32-34] developed by Kollberg et al. to facilitate the reconstruction of a writing episode. S-notation highlights the order in which revisions occur within a single document and represent the internal structure of writing and revising activities.

In S-notations, interruptions in the writing are noted by $\|_{i}$, where $i$ represents the order of the interruption. Insertions and deletions are marked with ${ }^{i}$ inserted text and ${ }^{i}$ (deleted text), respectively. Time spent paused in an interruption is noted as a superscript. If, after the pause, the writing resumes at that point, this is indicated by $\mid$ rather than $\|$. An example of this notation (modified from [32]) is

I am writing $a^{1}$ \{short $\}$ text $\|_{1}$. It will ${ }^{2}$ (probably) $\|_{3}^{15}$ be revised ${ }^{3}$ (somewhat) later. $\|_{2}$ Now ${ }^{4}\left(\mathrm{Iam} \|_{4}\right)$ it is ${ }_{5}^{10}$ finished.

The interpretation of this is as follows: First, the subject wrote "I am writing a text." Then, they interrupted their writing, as denoted by $\|_{1}$. This directs the reader to search for the matching superscript 1 , which is found earlier in the sentence; the use of the brackets in ${ }^{1}$ \{short $\}$ indicates that at this point the word "short" was inserted into the sentence. Writing resumes with the sentence "It will probably be revised somewhat later," at which point the writing is interrupted again $\left(\|_{2}\right)$ in order to delete the word "probably" and again, this time with a 15 second pause, $\left(\|_{3}^{15}\right)$ to delete the word "somewhat." Finally, they wrote the words "Now I am," then deleted the words "I am" and wrote "it is," paused for $10 \mathrm{~s}\left(\left.\right|_{5} ^{10}\right)$, and finally wrote the word "finished." The finished text is

I am writing a short text. It will be revised later. Now it is finished.

Software to both capture key strokes and automatically convert to S-notation was originally developed for Macintosh computers [32] and, subsequently, to parse temporal data from a simple web interface [35]. As both versions capture and report all revisions, including singleletter replacements (e.g., quick typos), the utility is a bit mixed. We have found it more useful to manually convert text to S-notation as part of the analysis process. That is, as we watch the essay's creation through replaying the keycapture data we note significant revisions and then add S-notation annotations to the text. The time spent doing so is approximately equal to that removing trivial revisions captured by automatic software and far more enlightening.

Of fundamental importance to this process, however, is that it is noninvasive to the writer (apart from the laboratory context). It is possible to conduct empirical studies of onthe-fly revision strategies without intruding on the writing process. In particular, we can subject the generation of discipline-specific content writing to the linguistic analysis of Schwartz, observing revisions of all types in real time, and attempt to infer their origin in student cognition.

\section{B. Context}

We now present a sample of data acquisition and initial analysis to illustrate the method. Previous work [36] investigated the use of different writing prompts in an introductory physics course for nonscience majors. From this work, a single prompt, involving buoyancy and density, was chosen as the focus of our pilot study. Our subject, Jesse (name changed), responded to a call for volunteers to engage in a writing exercise within the education research laboratory on a computer on which key-capture software was installed. Note that throughout this paper we intentionally conflate the mass and weight density, as the (admittedly important) distinction was neither relevant for the task at hand nor raised by the study subject.

At the time of our study, Jesse was a fourth year student majoring in Imaging Science. As part of this degree program he took a three-quarter sequence of calculus-based 
physics and received an average GPA of 2.6; it was assumed that Jesse would have a sufficient understanding of the topic to find the question challenging, but not impossible. This assumption is found to be justified by the progression of his essay.

The prompt used for this study was as follows:

$60 \mathrm{ml}$ of a mystery substances weighs $90 \mathrm{oz}$. Write a 250 word essay explaining, in detail, how you can use this information to predict the weight of $90 \mathrm{ml}$ of the substances in two ways: through the ideas of proportionality and density. Once the initial essay was complete, the student was instructed to revise the original, shortening it to 125 words.

A previous unpublished study found that this prompt elicited one of two fundamentally different responses. The first invokes proportional reasoning, with the most common manifestation articulating a ratio, mathematically realizing an often verbally stated reasoning " $60 \mathrm{ml}$ is to $90 \mathrm{oz}$ as $90 \mathrm{ml}$ is to $W^{\prime \prime}$ :

$$
\frac{60 \mathrm{ml}}{90 \mathrm{oz}}=\frac{90 \mathrm{ml}}{W} .
$$

The typical response then invokes algebra to solve for the missing weight, $135 \mathrm{oz}$. A related approach also involves proportional reasoning, but omits an explicit mention of a ratio, e.g., " $90 \mathrm{ml}$ is one-and-a-half times more than 60 , so the new weight is one-and-a-half times more than 90," or 135. Students often reported these as two separate techniques, not recognizing the mathematical similarity. We admit not knowing whether students truly did not see the similarity or were merely trying to repackage an old idea as something new.

The second method does not directly involve proportionality, but rather finds the weight of $1 \mathrm{ml}$ of the substance ("if $60 \mathrm{ml}$ weighs $90 \mathrm{oz}$, then $1 \mathrm{ml}$ weighs $60 / 90=$ $1.5 \mathrm{oz}$ ") and then uses that information to find the new weight ("if $1 \mathrm{ml}$ weighs $1.5 \mathrm{oz}$, then $90 \mathrm{ml}$ weighs $90 \times 1.5=135 \mathrm{oz}$ "). Operationally, this defines the material density, although this is rarely articulated explicitly (e.g., "the density would be weight (volume) or $90 / 60=1.5 \mathrm{oz} / \mathrm{ml}$, then the new weight would be volume times density, or $90 \times 1.5=135 \mathrm{oz}$ "). Recognizing this quantity as the density was the least likely approach, and so we consider it a rare variation of the method.

\section{ANALYSIS OF DISCOURSE}

Jesse's writing is highly nonlinear, with long pauses that lead to revisions elsewhere in the document. In order to interpret a given revision or piece of writing, one must keep in mind the entire context of the piece. Our presentation is as follows. First, we describe Jesse's writing without interpretation or analysis, noting only pauses and insertions or deletions. The writing occurs in 6 discrete intervals, each suggestive of potential for insight into Jesse's thinking. We then present Jesse's essay in S-notation, illustrating it's utility as a representational form. Finally, we present initial interpretations of Jesse's essay as suggested by the dynamic behavior. Our goal is not to claim definitive knowledge about Jesse's cognitive activity. Triangulation activities needed to support such claims could take the form of individual interviews, using the various stages of the essay as prompts for discussion, or a larger- $N$ study of the writing of larger numbers of students to look for common behavior. Rather, we try to draw attention to the new types of questions that the method of key capture raises.

\section{A. Jesse's writing of the essay}

In response to the given prompt, Jesse thought for a few seconds and then wrote.

I don't know anything about density; however, there are at least a couple ways of determining it or at least I'm told.

He then paused for 20 seconds and continued

The simplest way of determining it would be through the use of proportionality. In the example, a sample of $60 \mathrm{ml}$ of substance weighed 90 ounces. The question asks to determine how much $90 \mathrm{ml}$ of the same substance would weigh. To set this up, one must

At this point there is another pause of 18 seconds, before Jesse continues with

set up a ratio. So $60 \mathrm{ml}$ over 90 ounces is equal to $90 \mathrm{ml}$ over $x$ ounces. By cross multiplying and solving for $x$, one can find the value of $x$, which is the weight of the $90 \mathrm{ml}$ of substance.

After another 20 second pause, Jesse does not resume writing where he left off. Rather, he goes back and erases his first sentence ("I don't know anything about density"), and composes a new introductory paragraph:

Density is an important part of modern science, finding its way into many fields including chemistry, optics, and physics. Typically, if given a total amount of substance and a weight, one can find the weight of a different total amount of the same substance.

This is followed by another long (40 second) pause before concluding the new introductory paragraph:

One may also be able to use formulas to calculate a density by plugging numbers into a formula, but what it all boils down to, in this case, is the total amount of "stuff" within a specific volume. 
After this, Jesse returns to the immediate question of using the concept of density, pausing for about 20 seconds. He first modifies the first sentence of his second paragraph, removing the qualifying "or so I'm told" clause and then finishes the paragraph with

Another way of determining the total weight for the system would be to simply divide 90 ounces by $60 \mathrm{ml}$. Density is defined as mass over volume, so by doing this one has found the density. Once the density has been found, a person simply must multiply this number by a new given amount to find the new mass. This is the same procedure as before, but practiced a different way.

\section{B. S-notation}

In S-notation, Jesse's essay looks as follows. For convenience, we boldface the first words actually written. (Recall that text within brackets are later insertions, and so the entire first paragraph and first sentence of the second paragraph are not, in fact, where the writing begins. The superscripts 2 and 3 before the first sentence of each paragraph indicates that these were the second and third modifications.)

${ }^{2}\{$ Density is an important part of modern science, finding its way into many fields including chemistry, optics, and physics. Typically, if given a total amount of substance and a weight, one can find the weight of a different total amount of the same substance. $\left.\right|^{40}$ One may also be able to use formulas to calculate a density by plugging numbers into a formula, but what it all boils down to, in this case, is the total amount of "stuff" within a specific volume. $\left.\|_{3}^{20}\right\}$

${ }^{3}\{$ There are at least a couple ways of determining the weight of a substance when its mass and volume are known. $\|_{4}{ }^{1}(\boldsymbol{I}$ don't know anything about density; however, there are at least a couple ways of determining it or at least I'm told. $\left.\|_{2}\right)\left.\right|^{20}$ The simplest way of determining it would be through the use of proportionality. In the example, a sample of $60 \mathrm{ml}$ of substance weighed 90 ounces. The question asks to determine how much $90 \mathrm{ml}$ of the same substance would weigh. To set this up, one must $\left.\right|^{18}$ set up a ratio. So $60 \mathrm{ml}$ over 90 ounces is equal to $90 \mathrm{ml}$ over $x$ ounces. By cross multiplying and solving for $x$, one can find the value of $x$, which is the weight of the $90 \mathrm{ml}$ of substance. $\|_{1}^{20}$ ${ }_{4}^{4}$ Another way of determining the total weight for the system would be to simply divide 90 ounces by $60 \mathrm{ml}$. Density is defined as mass over volume, so by doing this one has found the density. Once the density has been found, a person simply must multiply this number by a new given amount to find the new mass. This is the same procedure as before, but practiced a different way.\}

\section{Sample analysis}

A rich narrative can be inferred from Jesse's six episodes that raise many potential research questions. To understand each, we place it in the context of the general prompt and the immediate task, drawing upon experience with prior student responses [36]. We emphasize the location and length of pauses, paying close attention to the writing that immediately precedes and follows.

\section{Initial response}

The prompt poses a specific question and ends with a request designed to prime a response in the context of proportionality and density. Prior research on attention (recounted in Kahneman [37]) has shown that readers often focus on the last words in a text, and we see an indication of this in Jesse's writing. Rather than set the context for the problem, by either repeating the details or placing density within a larger context, Jesse focuses on the last word of the prompt (density) and reports that he knows nothing about this particular concept. The nature of Jesse's initial writing suggests that much of what follows will be generative in nature. It appears that Jesse does not enter into the writing process with a firm idea of where he will end up and will be "making it up" as he goes, not merely regurgitating memorized information. Followup interviews could probe how Jesse perceives this activities and how he activates buried resources and knowledge to develop them into a coherent picture. Since reactivation and sense making are fundamental to the learning process [38], interesting research questions surround how this proceeds in a purely self-directed activity such as writing, without any direct teacher intervention or even presence.

\section{Proportionality}

Jesse pauses for twenty seconds, a rather long wait time, and thinks about how to proceed. Upon emerging from this think, Jesse again echoes the last words of the prompt ("proportionality") but now includes language to indicate that he can conceive of applying this concept to solve the problem and, indeed, that it is the "simplest" way of solving the problem. In doing so, Jesse is consistent with behaviors observed previously [36] in a class of nonscience majors, the majority of whom initially also choose a proportionality argument.

Jesse repeats salient numerical information from the prompt and repeats the question with the explanatory text of "The question asks..." and ends the episode midsentence with the fragment "To set this up, one must." We might infer, from this and the subsequent pause, that Jesse does not begin this episode with a clearly defined plan of action. An intriguing question is whether the consolidation of information in his own words, grouping the quantitative information in a spatially localized area, serves the purpose of conventional first steps of traditional problem solving of 
assembling relevant variables (e.g., GOAL [39] or Polya [40]). Given the subsequent (rather long) pause of $18 \mathrm{sec}-$ onds, triangulating methods are needed to determine whether this episode represents an advance in Jesse's understanding.

\section{First attempt at solution}

The third episode contains a complete explication of the proportional reasoning solution. It is a rapid-fire burst of information, containing technical terms not included in the prompt ("ratio"), a verbal description of what this ratio literally looks like ("60 ml over 90 ounces"), and instructions on the mathematical operations ("cross multiplying") required to solve the problem. Jesse's thinking begins to become more abstract, introducing variables $(x)$ with little obvious connection to the actual problem description. The choice of $x$ over a more representational symbol (e.g., $m$ ) suggests a formal mathematical frame [41], repeating an operation mechanically. The abstract variable is connected back to the problem, however, with the subjunctive clause describing it as "the weight of the $90 \mathrm{ml}$." The rapid putting of thought to paper, immediately following a pause, is suggestive of some form of cognitive reorganization. Little time is spent checking or reviewing this writing or thought process, either during or immediately after the writing, suggesting an understanding that is complete and thorough.

\section{A dramatic interruption}

After a 20 second pause, Jesse resumes writing not where he left off but, rather, returns to the very beginning of the narrative and erases the first sentence ("I don't know anything about density..."). It is this discontinuity in both time (a pause) and location that key-capture analysis excels at revealing, and a number of interesting research questions arise as a result. For example, while it appears that Jesse now realizes that he does, in fact, know (or remember) something about density, what exactly brings about this revision? Is the revision an attempt to reframe the essay and, if so, from what to what? Following Schwartz [26], we might classify this revision as a reformulation of structure, changing the essay's tone, although it may also contain elements of content reassessment, with Jesse now thinking of density in a new light.

Jesse's new introduction is in a decidedly different tone than the original essay. It is more formal and grandiloquent, with subjective declarations (density is "important" and finds its way into "many" fields). Contrasted with the previous specific descriptions of procedure (cf. episode 2), Jesse also describes how the concept of density can be used in generic situations, writing,

Typically, if given a total amount of substance and a weight, one can find the weight of a different total amount of the same substance.
Discourse analysis directs attention toward transitional words, and so questions are raised by the qualifying adverb "typically." Subsequent interviews might focus on how this situation is typical or what an atypical situation might look like. Jesse himself hints at this in the qualifying clause that the problem requires "the same substance" be used.

\section{Temporal markers in writing}

One argument we wish to advance in this paper is that key-capture research, with associated analysis, draws the researchers attention to writing events that might otherwise go unnoticed. An example is the single sentence that comprises what we have segregated into a separate episode. The episode begins with a 40 second pause, the longest in the entire writing sample at almost twice as long as any other pause. The writing does not change spatial location, remaining in the new introductory paragraph, and so, without key-logging data, we might assume, reasonably but erroneously, that the paragraph's conclusion was composed simultaneously with the rest of the paragraph. The pause draws attention to the concluding sentence, however, and there are other indications that this sentence might represent an important event in Jesse's composition. First, we notice that Jesse concludes by forgoing a mathematical definition of density and instead using the canonical concept of "amount of matter within a specific volume." Next, we see Jesse articulating the idea that a conceptual understanding might be more important than just the operational use of "plugging numbers into a formula," and the colloquial phrase "what it all boils down to" is in marked contrast with the more stilted language that comes earlier ("Density is an important part..."). The long pause and sudden change in writing tone both encourage the researcher to probe further this particular episode.

\section{Wrapping things up}

After a final 20 second pause, Jesse concludes by rapidly providing a second method for solving the problem. The ensuing text occurs in a single burst:

Another way of determining the total weight for the system would be to simply divide 90 ounces by $60 \mathrm{ml}$. Density is defined as mass over volume, so by doing this one has found the density. Once the density has been found, a person simply must multiply this number by a new given amount to find the new mass. This is the same procedure as before, but practiced a different way.

We call attention to the qualifier "simply," which Jesse now uses twice, the first appearance of a formulaic or semioperational definition (operationalized by the data from the preceding sentence) of density, and the final sentence, which seems to indicate that Jesse has succeeded in recognizing that the use of the concept and formula for 
density in fact reduces to the previously presented proportionality argument.

\section{Distinctive features of key-capture analysis}

In the absence of additional triangulating evidence, the inferences about Jesse's thinking in the preceding analysis - the generative nature of the response, the motivation behind the initial organization of ideas, and the specific meaning behind the essay's framing-remain unproven. Nevertheless, this analysis highlights two distinctive cognitive activities that would otherwise go unnoticed: the temporal inhomogeneity of exposition and holistic shift in framing that provides, in a hermeneutical sense, indication of cognitive evolution.

\section{Temporal inhomogeneity of exposition}

It is not surprising that writing occurs in bursts, with pauses indicating the need for thought. By highlighting the location and duration, key-capture analysis allows us to make specific assertions about how the author's thoughts proceed. For example, the pauses after the first, rather superficial, introductory sentences that make up episode 1 and the tentative start of episode 2 are hallmarks of an onthe-fly generative state. Compared with the rapid exposition in episode 3 , where a fully developed idea is written all at once, we see initial hesitancy leading to confidence within the span of 2 paragraphs. None of this is evident from the static final draft.

The idea that pauses indicate some organization of thoughts is supported by the pause before episode 6 . Again a pause is followed by a single burst of text containing a fully formed idea. Key-capture analysis reveals that the two different approaches are well separated temporally, with almost 2 full minutes (and much composition in a different part of the essay) elapsing between the end of episode 3 and the beginning of episode 6. This is compelling evidence that the two approaches were activated separately, and not initially related by Jesse to one another. An analysis of the static, final document would not reveal this. In fact, the spatial adjacency of the two descriptions would (erroneously) suggest that the writing of one had immediately followed the other. While keycapture analysis cannot determine if the two conceptual approaches were "learned" or "remembered," it does highlight the separate nature of Jesse's initial understanding.

\section{Holistic shift in framing}

It is tempting, in the absence of contradictory evidence, to assume that a text is written in the order in which it is read. As the previous sub-section illustrates, the ensuing assumption that two spatially proximate ideas are similarly linked in the author's mind can be misleading. Key-capture analysis also reveals temporally proximate but spatially distant events that are missed by static text analysis, and these also can give insight into the author's mind. The singular example of this is episode 4, where Jesse stops writing in the middle of his second paragraph and resumes by rewriting his initial sentences. This is a dramatic change in the framing of the essay, from a very specific, informal tone ("I don't know anything about density; however there are at least a couple ways of determining it or at least I'm told") to a more general, formal statement ("Density is an important part of modern science ..."). Key-capture analysis cannot answer questions about how closely these words mirror Jesse's thoughts (e.g., does he really initially feel like he knows nothing about density?), but these are not needed to make the plausible conclusion that something has changed in how Jesse views the essay. This shift, which appears significant, would be completely missed by a static textual analysis, which would only have the "final state" to go by.

Furthermore, key-capture analysis allows one to identify approximately when it changed, coming as it did after the first solution was described but before the second. The specifics of how the particular prompt inspired this change or how Jesse's thoughts transitioned from the initial to the final state cannot be learned without further triangulating research. Nevertheless, the use of key-capture analysis extends the types of information we can learn about student thinking from an essay's composition.

\section{CONCLUSION}

We have presented a research methodology that combines technologically available key-capture software with a revision-centric analysis format, S-notation, that highlights the discontinuous nature of writing and raises new questions about its use. We apply the method to a disciplinary context, an introductory physics prompt, and show that a single writing episode may be characterized with both long temporal pauses and jumps in the location of added or edited text. These phenomena cannot be observed with conventional writing research techniques, and so keycapture discourse analysis allows the researcher to identify promising rhetorical moves in a composition to pursue further. Most critically, the method is noninvasive, easily administered in a lab environment or (with appropriate ethical safeguards) on students' personal computers.

The promise of this future research is immense, as writing assignments have the promise of inducing significant student reflection with little or no explicit instructor interaction. While many claims have been made about the educational potential of reflective writing, we argue that real-time analyses of writing episodes can demonstrate possible mechanisms for how such learning might occur. Combined with traditional interviews, particularly with the replayed essay as a prompt, this technique allows a unique view into the student state of mind while writing is occurring, opening up new lines of questioning and research. 


\section{ACKNOWLEDGMENTS}

We gratefully acknowledge the members of RIT's Science \& Mathematics Education Research Collaborative for their careful reading of the manuscript.

[1] A. N. Applebee, Writing and reasoning, Rev. Educ. Res. 54, 577 (1984).

[2] J. Emig, Writing as a mode of learning, College Comp. Commun. 28, 122 (1977).

[3] Language connections: Writing and reading across the curriculum, edited by T. Fulwiler and A. Young (National Council of Teachers of English, Urbana, IL, 1982), pp. ix-xiii.

[4] C. H. Knoblauch and L. Brannon, Writing as learning through the curriculum, College English 45, 465 (1983).

[5] S. H. McLeod, Writing across the curriculum: The second stage, and beyond, College Comp. Commun. 40, 337 (1989).

[6] L. Odell, The process of writing and the process of learning, College Comp. Commun. 31, 42 (1980).

[7] A. J. Herrington, Writing to learn: Writing across the disciplines, College English 43, 379 (1981).

[8] C. Bazerman, Reference Guide to Writing Across the Curriculum, Reference guides to rhetoric and composition (Parlor Press, West Lafayette, IN, 2005).

[9] C. Bazerman and D. R. Russell, Landmark Essays on Writing Across the Curriculum, Landmark Essays on Writing Across the Curriculum No. v. 6 (Hermagoras Press, Davis, CA, 1994).

[10] A. Young and T. Fulwiler, Writing across the disciplines: research into practice (Boynton/Cook Publishers, Portsmouth, NH, 1986).

[11] C. Anson, Writing to learn versus learning to write, Proceedings of the Seventh National WAC Conference, St. Louis, Missouri, 2004.

[12] J. M. Ackerman, The promise of writing to learn, Written Commun. 10, 334 (1993).

[13] P.D. Klein, Reopening inquiry into cognitive processes in writing-to-learn, Educ. Psychol. Rev. 11, 203 (1999).

[14] T. L. Hein, Using student writing as a research and learning tool, AAPT Announcer 27, 79 (1998).

[15] T. L. Hein, Writing: An effective learning tool for nonscience majors, AAPT Announcer 29, 114 (1999).

[16] D. T. Brookes and E. Etkina, Using conceptual metaphor and functional grammar to explore how language used in physics affects student learning, Phys. Rev. ST Phys. Educ. Res. 3, 010105 (2007).

[17] M. Ediger, Writing in the mathematics curriculum, J. Instr. Psych. 33, 120 (2006).

[18] R. A. Ellis, University student approaches to learning science through writing, Int. J. Sci. Educ. 26, 1835 (2004).

[19] M. K. Florence and L. D. Yore, Learning to write like a scientist: Coauthoring as an enculturation task, J. Res. Sci. Teach. 41, 637 (2004).
[20] E. W. Patterson, Structuring the composition process in scientific writing, Int. J. Sci. Educ. 23, 1 (2001).

[21] W. A. Sandoval and K. A. Millwood, The quality of students' use of evidence in written scientific explanations, Cognit. Instr. 23, 23 (2005).

[22] L. D. Yore, B. M. Hand, and M. K. Florence, Scientists' views of science, models of writing, and science writing practices, J. Res. Sci. Teach. 41, 338 (2004).

[23] R. L. Bangert-Drowns, M. M. Hurley, and B. Wilkinson, The effects of school-based writing-to-learn interventions on academic achievement: A meta-analysis, Rev. Educ. Res. 74, 29 (2004).

[24] L. Flower and J. R. Hayes, A cognitive process theory of writing, College Comp. Commun. 32, 365 (1981).

[25] L. Faigley and S. Witte, Analyzing revision, College Comp. Commun. 32, 400 (1981).

[26] M. Schwartz, Two journeys through the writing process, College Comp. Commun. 34, 188 (1983).

[27] J. Fitzgerald, Research on revision in writing, Rev. Educ. Res. 57, 481 (1987).

[28] L. Beason, Feedback and revision in writing across the curriculum classes, Res. Teach. Engl 27.4, 395 (1993).

[29] C. M. Anson and R. A. Schwegler, Tracking the mind's eye: A new technology for researching twenty-first-century writing and reading processes, College Comp. Commun. 64.1, 151 (2012).

[30] M. Leijten and L. Van Waes, Keystroke logging in writing research: Using inputlog to analyze and visualize writing processes, Written Commun. 30, 358 (2013).

[31] http://www.inputlog.net.

[32] P. Kollberg, S notation as a tool for analyzing the episodic structure of revisions, (1996), European Writing Conferences, ftp.nata.kth.se/iPLab/TechReports/IPLab123.ps.

[33] Kolberg, Ph.D. thesis, Royal Institute of Technology, 1998.

[34] E. K. Severinson and P. Kollberg, A computer tool and framework for analyzing on-line revisions, in The science of writing (Lawrence Erlbaum Associates, Hillsdale, NJ, 1996).

[35] D. Demaree, Ph.D. thesis, The Ohio State University, 2006.

[36] L. Hermsen and S. V. Franklin, A new research agenda for writing-to-learn: embedding cognition in discipline, in Deaf Cognition, edited by M. Marschark and P. C. Hauser (Oxford University Press, New York, 2005), Chap. 15.

[37] D. Kahneman, Thinking, Fast and Slow (Farrar, Straus and Giroux, New York, 2011). 
[38] A. Karelina and E. Etkina, When and how do students engage in sense-making in a physics lab, in Proceedings of the Physics Education Research Conference 2006, PER Conference, Vol. 883 (Syracuse, New York, 2006), pp. 93-96.

[39] R. Beichner, D. Deardorff, and B. Zhang, GOAL-oriented Problem Solving, Tech. Rep. (1999).
[40] G. Polya and J. H. Conway, How to Solve It: A New Aspect of Mathematical Method, Princeton Science Library (Princeton University Press, Princeton, NJ, 2014).

[41] D. Tannen, Framing in Discourse, Framing in Discourse (Oxford University Press, New York, 1993). 